\title{
PERSONNA: proposta de ontologia de contexto e perfil de aluno para recomendação de objetos de aprendizagem
}

PERSONNA: a proposal of student's context and profile ontology for learning objects recommendation

Paulo Rezende

Programa de Pós-graduação em

Ciência da Computação

Universidade Federal de

Juiz de Fora (UFJF)

paulo.rezende@ead.ufjf.br

José Maria N. David

Programa de Pós-graduação em

Ciência da Computação

Universidade Federal de

Juiz de Fora (UFJF)

jose.david@ufjf.edu.br
Crystiam Kelle Pereira

Programa de Pós-graduação em

Ciência da Computação

Universidade Federal de

Juiz de Fora (UFJF)

crystiam.kelle@ead.ufjf.br

Regina Braga

Programa de Pós-graduação em

Ciência da Computação

Universidade Federal de

Juiz de Fora (UFJF)

regina.braga@ufjf.edu.br

\author{
Fernanda Campos \\ Programa de Pós-graduação em \\ Ciência da Computação \\ Universidade Federal de \\ Juiz de Fora (UFJF) \\ fernanda.campos@ufjf.edu.br
}

\begin{abstract}
Resumo Um sistema de recomendação na área educacional tem por objetivo monitorar dados do aluno quando este interage com aplicações educacionais, para que seja possível sugerir objetos de aprendizagem adequados às suas preferências. Este trabalho descreve uma ontologia denominada PERSONNA para modelagem do perfil e do contexto do aluno em um ambiente e-learning, a fim de padronizar as informações e facilitar o processo de recomendação de objetos de aprendizagem em uma arquitetura específica, denominada BROAD-RS.
\end{abstract}

Palavras-Chave: Ontologia, Contexto, Perfil, Objetos de Aprendizagem, Sistema de Recomendação.

\begin{abstract}
A recommendation system in the educational area aims to track student data when interacting with educational applications to suggest appropriate learning objects. This paper describes an ontology named PERSONNA to support modeling of student's profile and context in an elearning environment in order to standardize information and facilitate the process of recommending learning objects in BROAD-RS architecture.
\end{abstract}

Keywords: Ontology, Context, Profile, Learning Objects, Recommendation System. 


\section{Introdução}

Para que um sistema computacional possa recomendar objetos de aprendizagem (OA) a um aluno, de forma individual e personalizada, é necessário que o sistema conheça suas características, ou seja, o sistema deve obter e gerenciar algumas informações básicas sobre o perfil e o contexto do aluno. Dentre elas, cabe ressaltar suas preferências, seus objetivos e estilos de aprendizagem. Além disso, estas informações devem estar padronizadas a fim de evitar inconsistências semânticas e facilitar a interoperabilidade entre os diversos módulos e sistemas [12].

Com o objetivo de estabelecer um padrão que seja essencial ao intercâmbio dessas informações, organizações internacionais como Institute of Electrical and Electronic Engineers-IEEE (https://www.ieee.org/), Instructional Management Systems-IMS (http://www.imsglobal.org/) e Advanced Distributed Learning-ADL (www.adlnet.org/) trabalham na padronização de informações de ambientes de aprendizagem, especificando metamodelos de dados para informações sobre alunos [12].

Os padrões disponíveis na literatura apresentam uma grande diversidade de elementos e abordagens, o que compromete a interoperabilidade dos dados. A falta de padronização nas instâncias gera diversos problemas, com destaque para a falta de confiabilidade, integridade e qualidade desses dados. Além disso, o suporte a múltiplas visões dos dados, protocolos de comunicação e definição de repositórios para armazenamento desses dados não fazem parte do escopo dos padrões estudados. Um modelo compacto e flexível, que privilegie a semântica das informações e a padronização dos conceitos pode contribuir para o seu reuso nas aplicações na área educacional.

As limitações observadas motivaram o desenvolvimento de um modelo conceitual, que forneça os principais dados sobre o aluno, e que possa ser utilizado em sistemas de recomendação. Assim, o objetivo deste artigo é apresentar uma ontologia de modelagem do perfil e do contexto do aluno, denominada PERSONNA, que busca padronizar as informações do aluno, considerando o contexto utilizado e assim facilitar o processo de recomendação de objetos de aprendizagem na arquitetura BROAD-RS [21].

Em [15], [17] e [25] são apresentadas propostas similares. No entanto, [17] é proposta uma modelagem de domínio através do uso de ontologias para educação mas que não contempla conhecimento específico em engenharia de conhecimento, o que pode comprometer sua utilização para uso em sistemas de recomendação, cujo foco deve ser também no uso de mecanismos inteligentes para o processamento de informações. Em [15] é apresentada uma abordagem baseada em ontologias para anotação automática de OA. Considerando o contexto da PERSONNA, esta abordagem difere um pouco considerando que não considera a evolução do perfil do usuário e nem privilegia informações de contexto. $O$ trabalho de Sosnovsk et al. [25] engloba a modelagem de usuários e ontologias web no contexto de sistemas adaptativos. No entanto, não possui foco na modelagem de perfil e contexto.

O projeto BROAD pesquisa a adoção de tecnologias como ontologias, serviços web semânticos, agentes e workflows numa arquitetura que personalize a recomendação de objetos de aprendizagem. Nesse contexto, a ontologia PERSONNA avança em relação à base de conhecimento semântico (contexto e perfil de alunos) usada em trabalhos prévios do grupo [2] [5] [23] [4] [26]. Além de servir como base para modelagem do perfil e contexto do aluno, filtra as informações relevantes do aluno de acordo com o contexto de localização e facilita o processo de recomendação de objetos de aprendizagem da arquitetura BROAD-RS.

O presente trabalho está organizado da seguinte forma: na seção 2 são apresentados os principais padrões educacionais, que fundamentam este trabalho; na seção 3 são detalhadas algumas propostas de recomendação e é realizada uma análise comparativa das propostas, a seção 4 descreve a ontologia proposta e suas especificações; a seção 5 apresenta a arquitetura BROAD-RS e a utilização da ontologia PERSONNA, e, por fim, a seção 6 oferece as conclusões do estudo e suas perspectivas futuras.

\section{Especificações/Padrões de Alunos}

Considerando o contexto educacional, existem na literatura, diversas propostas para a especificação e padronização de dados sobre contexto e perfil do aluno. Dentre as principais especificações de dados educacionais podem ser citadas: o EduPerson [8], o ULF (Universal Learning Format) [22], o PAPI (Personal and Private Information), da IEEE [19] e o IMS LIP (Instructional Management Systems Learning Information Package), da Global Learning Consortium [14].

EduPerson [8] foi desenvolvido com o objetivo de facilitar a comunicação entre sistemas de instituições de ensino superior. O objetivo era definir uma classe de objetos para descrever pessoas em universidades e para ser utilizada em diretórios LDAP (Lightweight Directory Access Protocol). O grupo de trabalho também definiu recomendações sobre a semântica, a sintaxe e a privacidade dos dados. A versão 1.6 do EduPerson [8] possui 42 atributos divididos em duas categorias: os atributos gerais (atributos comuns como nome, e-mail, endereço, entre outros) e os atributos novos, que foram criados para facilitar a colaboração interinstitucional das aplicações. Cada 
atributo tem uma classe utilitária de aplicação associada, a qual sugere a classe de aplicações para as quais esse atributo é apropriado.

O ULF (Universal Learning Format) [22] é um conjunto de formatos baseados em XML (Extensible Markup Language). Possui o objetivo de fornecer portabilidade para dados educacionais, sendo que os formatos que o compõem podem ser mapeados para outros padrões. O formato é composto por cinco elementos: CatalogFormat (Formato Catálogo), Learning Content Format (Formato de Conteúdo Educacional), Competency Format (Formato de Competência), Certification Format (Formato de Certificação) e Profile Format (Formato do Perfil).

A especificação PAPI (Public and Private Information Learner) [19] originou-se em 1998 no grupo IEEE Learning Technology Standards Committee (LTSC). Porém, em 2001, o seu desenvolvimento foi transferido para o Working Group Subcommittee que desenvolve padrões internacionais de dados de aluno para a ISO/IEC (Internacional Organization for Standardization / InternationalElectrotechnicalCommission) [19]. O PAPI especifica a sintaxe dos dados do aluno caracterizando suas informações pessoais, seus conhecimentos, preferências, entre outras [19]. A sua principal característica é a divisão das informações em seis categorias principais, que visam organizar os diversos tipos de dados do aluno. No documento PAPI são descritas regras para codificação dos elementos de dados para XML ou DNVP (Dotted Name-Value Pair).

A especificação LIP (Learner Information Package) [14] foi definida pelo Global Learning Consortium da IMS (Instructional Management Systems), que é um consórcio de instituições educacionais, companhias de software e editoras. O objetivo do consórcio é promover a disseminação de especificações que permitam a construção de ambientes de aprendizagem distribuídos [14]. De 1997 a 1999, a IMS trabalhou em conjunto com o grupo LTSC na definição do PAPI [19], porém no final de 1999, IMS decidiu produzir sua própria especificação para dados de alunos. Finalmente, em 2001 foi produzido o documento LIP que inclui o modelo de dados e esquemas XML para implementação do modelo.

Considerando os padrões descritos acima, é possível perceber a diversidade de elementos e da abrangência de cada padrão. Além disso, verifica-se que a interoperabilidade é um importante requisito ainda não totalmente atendido. Os padrões definem o conjunto de dados necessário, porém o domínio de cada dado nem sempre é explicitado. Os padrões definem a maioria dos campos como do tipo texto, o que permite inserir texto livre. Isso é um das grandes limitações para a interoperabilidade, pois a falta de padronização nas instâncias gera diversos problemas, por exemplo, um registro sobre o desempenho do aluno deve ter uma classificação padrão, pois um sistema pode representar notas sobre uma atividade usando uma classificação de 1 a 10 , enquanto que outro sistema pode utilizar conceitos como A, B ou C.

É possível destacar que a comunidade de pesquisa da área reconhece a importância de se definir um modelo padrão de dados de aluno que possa ser compartilhado entre diversos sistemas. Sendo assim, a tabela 1 apresenta a relação entre as categorias PAPI [19], LIP [14] e ULF [22].

\begin{tabular}{l|l|l}
\hline \multicolumn{1}{c|}{ PAPI [19] } & \multicolumn{1}{c|}{ LIP [14] } & \multicolumn{1}{c}{ ULF [22] } \\
$\begin{array}{l}\text { Personal } \\
\text { Information }\end{array}$ & Identification & Personal \\
\hline $\begin{array}{l}\text { Relations } \\
\text { Relationships } \\
\text { /affiliation }\end{array}$ & - \\
\hline $\begin{array}{l}\text { Pccessibility, } \\
\text { Interest }\end{array}$ & Preference \\
\hline Performance & $\begin{array}{l}\text { Transcript, goal, } \\
\text { qcl, activity }\end{array}$ & $\begin{array}{l}\text { Learning/ } \\
\text { Observation }\end{array}$ \\
\hline Portfolio & $\begin{array}{l}\text { Activity, } \\
\text { competency }\end{array}$ & $\begin{array}{l}\text { Competency } \\
\text { /Certification/ } \\
\text { Goal }\end{array}$ \\
\hline Security & Security key & - \\
\hline- & - & $\begin{array}{l}\text { Profile } \\
\text { Information }\end{array}$ \\
\hline
\end{tabular}

Tabela 1 - Relação entre categorias dos modelos.

As categorias Relations (Relacionamentos) (PAPI) e Affiliation (Afiliação) (LIP) descrevem os relacionamentos que o aluno possui, sendo essa informação não contemplada no ULF.

A categoria Affiliation (Afiliação) permite o registro de organizações pelas quais o aluno teve algum tipo de relacionamento (aluno, funcionário, entre outros), bem como o período desse relacionamento. Essa categoria entra em conflito com outras categorias do próprio LIP como: Activity (Atividade), Competency (Competências) e QCL (Qualificações, Certificados e Licenças), que também podem possuir esse tipo de informação. LIP também apresenta a categoria Relationship (Relacionamento), que serve apenas para mapear relacionamentos entre os tipos de categorias do LIP, como, por exemplo, uma instância de Relationship (Relacionamento) pode informar que um registro da categoria QCL tem relação com um determinado registro da categoria Transcript (Transcrição).

A categoria Preference (Preferências) do PAPI permite descrever qualquer tipo e informação relacionada com as preferências do aluno, tanto no nível de interesse pessoal quanto no nível de acessibilidade. Essas informações estão divididas em duas categorias no LIP: Accessibility (Acessibilidade) e Interest (Interesse). Accessibility (Acessibilidade) consiste apenas das preferências do aluno quanto a idiomas e questões técnicas. A categoria Interest (Interesses) do LIP contém as informações de interesse pessoal do aluno como seus hobbies, entre ou- 
tros. ULF possui a categoria Preference Information (Informação de Preferências) que permite qualquer tipo de informação relacionada com preferências do aluno. Uma das vantagens que o PAPI apresenta, nesse tipo de categoria, é a possibilidade de estabelecer prioridades entre as preferências.

Informações sobre qualificações, certificados, licenças, atividades realizadas, objetivos e competências estão referenciadas na categoria Performance (Desempenho) do PAPI. No LIP esse tipo de informação está separado por categoria: QCL (Qualificações, Certificados e Licenças), Activity (Atividade), Competency (Competências), Goal (Objetivos), Transcript (Transcrição). O mesmo ocorre no modelo ULF, esse tipo de informação está dividido entre as categorias Certification Information (Informação de certificado), Competency Information (Informação de competência) e Goal Information (Informação de objetivo).

Os critérios: segurança, privacidade, extensibilidade, flexibilidade e suporte a implementação, também foram considerados para comparação dos modelos. Quanto à segurança e privacidade dos dados o PAPI define alguns mecanismos para prover segurança e privacidade. O LIP permite a inclusão de mecanismos para manter a privacidade e proteger a integridade dos dados. O padrão ULF não trata da questão de privacidade e segurança, que são itens importantes para assegurar um intercâmbio de dados consistente e seguro.

Quanto à extensibilidade e flexibilidade, os padrões permitem a inclusão de outros tipos de informação, possibilitando a sua extensão. Quanto ao suporte para o processo de implementação do padrão, o PAPI [19] apresenta um documento que explicita o mapeamento do modelo conceitual para XML. O LIP apresenta o XML Binding que descreve o processo de codificação dos dados e um guia para a implementação do padrão. A Tabela 2 apresenta o comparativo realizado nesses itens entre os modelos PAPI [19], IMS LIP [14] e ULF [22].

\begin{tabular}{l|l|l|l} 
& PAPI [19] & LIP [14] & ULF [22] \\
\hline Segurança & $\begin{array}{l}\text { Parcial- } \\
\text { mente }\end{array}$ & $\begin{array}{l}\text { Parcial- } \\
\text { mente }\end{array}$ & $\begin{array}{l}\text { Não } \\
\text { possui }\end{array}$ \\
\hline Privacidade & $\begin{array}{l}\text { Parcial- } \\
\text { mente }\end{array}$ & $\begin{array}{l}\text { Parcial- } \\
\text { mente }\end{array}$ & $\begin{array}{l}\text { Não } \\
\text { possui }\end{array}$ \\
\hline $\begin{array}{l}\text { Extensibi- } \\
\text { lidade }\end{array}$ & Plenamente & Plenamente & $\begin{array}{l}\text { Parcial- } \\
\text { mente }\end{array}$ \\
\hline $\begin{array}{l}\text { Suporte a } \\
\text { implementa- } \\
\text { ção }\end{array}$ & $\begin{array}{l}\text { Parcial- } \\
\text { mente }\end{array}$ & Plenamente & $\begin{array}{l}\text { Não } \\
\text { possui }\end{array}$ \\
\end{tabular}

Tabela 2 - Comparativo entre os modelos.

Em qualquer um dos padrões, o processo de identificação dos alunos é totalmente definido na implementação, sendo que não existe um modelo de confiabilidade, integridade e qualidade dos dados. Nenhum dos modelos define técnicas para validar a informação de desempenho e portifólio do aluno. Além disso, o suporte a múltiplas visões dos dados, protocolos de comunicação e definição de repositórios, para armazenamento desses dados, não faz parte do escopo de nenhum dos padrões.

Apesar do objetivo do EduPerson (EDUPERSON, 2005) ser a troca de dados de alunos entre instituições de ensino superior, os dados especificados no EduPerson apenas descrevem o aluno em termos gerais, como seus dados pessoais e instituições das quais ele faz parte. Dados sobre as suas preferências, objetivos, competências e desempenho em atividades, não são abordados. Outro ponto importante é que o EduPerson foi modelado para um contexto específico, ou seja, voltado para um ambiente de diretórios LDAP, onde a atualização constante desses dados não é facilitada.

Em geral, as propostas apresentadas dão ênfase na decomposição do modelo do aluno por características e não descrevem a metodologia utilizada para escolha dos, apenas as classificam baseados em sistemas que as implementam.

As características analisadas mostram que os padrões são bastante similares, porém cada um apresenta deficiências em algumas características. Descrever essas características na forma de uma ontologia e suas regras semânticas pode: facilitar seu uso em sistemas educacionais, buscar uma relação de denominação e de definição de termos precisa, permitir a integração dos modelos propostos, além de facilitar a descoberta de conhecimento e relações implícitas entre termos, a partir da especificação de regras ontológicas.

\section{Trabalhos Relacionados}

Nesta seção são apresentadas algumas propostas de arquiteturas, modelos e frameworks de recomendação e compartilhamento de informações de contexto que utilizam ontologias em suas soluções. O objetivo da catalogação destas propostas é identificar elementos de perfil e de contexto do aluno e que podem integrar a ontologia PERSONNA.

Brusilovsky e Peylo [3] apresentam um framework para compartilhamento de dados de alunos. O principal componente desse framework é um servidor de ontologias que é responsável por armazenar os modelos de aluno. $\mathrm{O}$ servidor de ontologias não desempenha qualquer modelagem do perfil do aluno, limitando-se a armazenar o seu nível de conhecimento para cada conceito da ontologia de domínio, adquirido por qualquer sistema que possua modelo de aluno. Os valores enviados por diferentes sistemas são armazenados separadamente no servidor. O servidor de ontologias pode ser composto em múltiplos servidores, desde que todos tenham o mesmo domínio. 
Cada servidor armazena uma ontologia específica e as informações sobre os conteúdos educacionais e dados sobre o perfil do aluno, são expressos em termos dessa ontologia. Quando vários sistemas decidem compartilhar e/ou trocar modelos, eles devem selecionar a ontologia desejada na troca. Após um sistema selecionar uma ontologia para modelar o aluno, o servidor de ontologias é capaz de conhecer o formato que deve enviar os dados sobre o aluno para esse sistema, assim como sabe onde e como requisitar dados sobre o aluno. Podemos destacar alguns itens que compõem a ontologia como objetivos, métodos, componentes e dados dos alunos.

Niederée [18] também propõe o uso de um modelo baseado em ontologias para a troca de dados de usuários entre sistemas. O modelo foi chamado de UUCM (Unified User Context Model), ou seja, modelo unificado de contexto e usuário e é a base para o processo de personalização. O modelo possui como vantagem o fato de permitir a construção de engines alteráveis de personalização de usuários, o que facilita a construção de ferramentas que permitem o usuário analisar e modificar a informação armazenada sobre ele. O modelo UUCM está focado no contexto do usuário, que é estruturado em diferentes dimensões e considera o fato de que o usuário interage com o sistema em diferentes contextos de trabalho. Para permitir a personalização, o sistema deve ser flexível e extensível o suficiente para lidar com as variações de abordagens relevantes na captura das características do usuário e sua situação atual. Podemos destacar as tarefas e relações com outros usuários, em função da mesma focar no contexto de trabalho do usuário.

Dolog e Nejdl [6] apresentam a contextualização e implementação de uma ontologia que oferece uma base comum para a troca de dados de modelos de alunos entre diversas fontes de dados. A ontologia é focada em dados sobre o desempenho do aluno e suas competências. Essas competências são avaliadas através dos testes realizados pelo aluno. No momento em que ele alcança alguma competência, o seu desempenho é alterado. A ontologia também compreende dados relacionados sobre as preferências do aluno como: idioma, dispositivos, recursos e preferências de estilo de aprendizagem. Sistemas que desejam trocar dados devem utilizar a ontologia como modelo. Se os sistemas possuírem dados representados em um modelo diferente do modelo de troca, eles devem oferecer o mapeamento entre eles. A ontologia proposta por Dolog e Nejdl [6] é baseada nos padrões PAPI [19] e LIP [14] e está centrada nos dados sobre o desempenho do aluno, seus objetivos e avaliações além de dados relacionados às preferências do aluno como: idioma, dispositivos, recursos e preferências de estilo de aprendizagem, o que a torna ampla e mais interoperável.

Sudhana et al. [27] descrevem uma proposta de recomendação de recursos educacionais que considera ele- mentos do contexto do aluno fazendo uso de ontologias para representar este contexto. As informações contextuais usadas são divididas como sendo parte do contexto estático ou do contexto dinâmico. No contexto estático são consideradas as informações pessoais (ex. nome, qualificação, nacionalidade) e as informações referentes ao ambiente educacional do aluno, que geralmente não estão sujeitas a constantes mudanças. Já no contexto dinâmico, são relevantes as informações relacionadas à preferência e ao interesse do aluno, que podem mudar durante o processo educacional. A arquitetura proposta por Sudhana et al. [27] é composta por três bases de dados: (i) base do perfil do aluno, (ii) base de atividades educacionais e (iii) base de conhecimento. O conteúdo da base de dados do perfil do aluno é coletado através do seu registro que contém dados pessoais como qualificação, posição, preferências, entre outros. $\mathrm{O}$ calendário e as atividades educacionais do aluno são armazenados na base de dados de atividades educacionais. A base de dados de conhecimento armazena informações contextuais, coletadas de diferentes fontes.

Alharbi et al. [1] descrevem uma arquitetura baseada em contexto para recomendação em PLEs (Ambientes Pessoais de Aprendizagem). O foco da recomendação desta proposta são serviços independentes condizentes com o interesse do usuário. A recomendação é feita a partir de informações contextuais dos serviços e do aluno. O contexto dos serviços é adquirido através de sensores virtuais, e o contexto do usuário considera as informações do seu perfil e seu feedback em relação a recomendações anteriores. As informações de contexto do usuário são categorizadas em pessoais ou estáticas e preferências. As informações pessoais são: nome, endereço, idade, estado civil e e-mail. O resultado da recomendação é um conjunto de serviços e recursos sugeridos a partir do interesse atual do usuário, gerando notificações de links e páginas. A arquitetura do PLE, sensível ao contexto, consiste em duas camadas: a camada do PLE e a camada provedora de serviços. A camada provedora de serviços é composta de várias ferramentas e prestadores de serviços independentes como, por exemplo, Facebook ${ }^{\circledR}$, YouTube ${ }$, calendário pessoal e a biblioteca digital IEEE Xplore ${ }^{\circledR}$. Ela fornece informações contextuais, através de sensores virtuais. Estas informações brutas são capturadas por APIs para interagir com a camada do PLE.

Klašnja-Milicevic et al. [16] propõem uma arquitetura de um sistema de recomendação baseado em tags para ambientes de e-learning, nomeado Protus. O Protus é um sistema de tutoria projetado para ajudar os alunos no aprendizado dos fundamentos de linguagem de programação. O principal objetivo é recomendar materiais úteis e interessantes para alunos à distância, tendo como base suas diferentes experiências, preferências, objetivos de 
aprendizagem e outros atributos significativos. A arquitetura proposta por Klašnja-Milicevic et al. [16] é composta do módulo learner-system interaction responsável pela coleta dos dados sobre as atividades dos alunos (como padrões sequenciais, páginas visitadas, resultados de testes e notas obtidas) e a construção do modelo do aluno, considerando essas informações. O módulo off-line usa o modelo do aluno, construído no módulo learner-system interaction, para reconhecer os objetivos dos alunos, seu estilo de aprendizagem, e para filtrar objetos de aprendizagem que possuam conteúdo apropriado a eles. O módulo Recommendation Engine produz uma lista de recomendação de objetos de aprendizagem. A partir dos objetos filtrados, são enviados recursos e ações recomendados, alterando a interação do aluno com o sistema. O Protus utiliza técnicas de "clusterização" para agrupar os alunos pelo seu estilo de aprendizagem. Após a definição dos grupos, eles são usados para determinar atividades educacionais apropriadas. Em seguida, uma lista de recomendações pode ser criada de acordo com tags de alunos e de especialistas para cada grupo gerado [16].

Wang e $\mathrm{Wu}$ [29] apresentam uma proposta de recomendação sensível ao contexto para implementar um sistema educacional ubíquo (u-leaning) adaptativo. O objetivo é permitir ao aluno construir o seu ambiente de forma auto gerenciada e personalizada. Os autores esclarecem que a proposta está baseada nas abordagens de inquiry-based learning, social learning e context learning theories. Usando as transmissões da rede sem fio e a detecção das metas de aprendizagem em situações reais, através de sensores, o material didático apropriado é recomendado à plataforma de aprendizagem do aluno, considerando o seu contexto. O método de recomendação adotado pelo estudo utiliza a experiência dos usuários próximos que possuem metas de aprendizagens semelhantes e a avaliação feita por eles aos recursos educacionais. Após a recomendação, o aluno avalia o item recomendado e aceito por ele. Este feedback dado pelo aluno permite o aprendizado do sistema e uma recomendação mais qualificada, uma vez que recursos bem aceitos e avaliados são recomendados, posteriormente, a outros alunos. A definição do perfil do aluno é feita usando a técnica de filtragem colaborativa [24], e mineração dos registros de navegação na Web (Web Usage Mining) [29].

Em Fritzen et al. [9] é apresentada uma proposta que busca modelar o contexto a partir da extração de mensagens, trocadas entre alunos em grupos de Redes Sociais, e usar esse contexto para melhorar a relevância na recuperação de documentos na Web, tendo como objetivo apoiar a aprendizagem colaborativa. A intenção da proposta é tornar a recuperação de informação sensível ao contexto das discussões (por intermédio do uso das mensagens para modelagem do contexto), oferecendo, portanto, resultados de busca contextualizada. Para a aquisição do contexto, os autores propõem a captura a partir do enriquecimento das mensagens em dados abertos e com essa técnica melhorar as consultas do usuário, fornecendo-lhe conteúdo mais adequado na Web [9]. De acordo com Fritzen et al. [9] duas macro funcionalidades podem ser identificadas na proposta: processamento do contexto e processamento da consulta do usuário. As mensagens do grupo fornecem conteúdo para o processamento do contexto, através da colaboração entre os usuários. Quando uma solicitação de consulta é identificada, inicia-se o processamento para extrair os termos sugeridos para expansão do contexto e geração de uma mensagem de resposta ao usuário que escolhe os termos para expandir a consulta e avalia os resultados da busca.

Dwivedi e Bharadwaj [7] apresentam uma proposta de recomendação baseada na avaliação de conteúdo em uma rede social educacional, onde os alunos compartilham uns com os outros as suas classificações para recursos, incluindo livros, materiais didáticos, entre outros. Sempre que um aluno deseja uma recomendação sobre um determinado conteúdo, ele envia uma consulta para o seu amigo imediato e para os amigos distantes. $\mathrm{O}$ amigo imediato é aquele que está ligado a ele por um salto (ligado diretamente), enquanto que o amigo distante é aquele que está ligado a ele por 2 ou mais saltos. Os alunos que usaram o recurso, no passado, respondem à consulta com suas classificações. Com base nas respostas e no histórico das classificações entre amigos, é feito o cálculo da pontuação para a recomendação. O projeto da rede social apresentada considera o estilo de aprendizagem e o nível de conhecimento de tal forma que os alunos que possuem certo grau de similaridade nesses atributos são considerados amigos imediatos. Sendo assim, a recomendação feita por um amigo imediato será compatível com o estilo de aprendizagem e o nível de conhecimento do aluno. A proposta considera ainda a possibilidade do amigo imediato não ter tido contato com o material, cujo aluno deseja uma recomendação. Nesse caso a consulta do aluno é propagada para os amigos distantes. Além disso, a proposta explora associações de transitividade dentro da rede. Um estudo experimental realizado mostrou que a precisão das recomendações baseadas em recuperação associativa é significativamente melhor do que a tradicional filtragem colaborativa.

Em Gaeta et al. [17] é proposta uma modelagem de domínio através do uso de ontologias para educação. Nela é descrito com detalhes técnicas para construir ontologias de e-learning e como elas podem ser exploradas, a fim de definir e representar experiências em e-learning e gerenciar seu ciclo de vida, sem qualquer conhecimento específico em engenharia de conhecimento. Os autores 
definem uma abordagem que permite a colaboração e gerenciamento do conhecimento na construção e manutenção de ontologias. O foco na definição de ontologias por não especialistas em engenharia de conhecimento como professores, tutores entre outros, contribui para o a definição de modelos de experiências personalizadas de e-learning considerando as preferências e características cognitivas dos alunos. No entanto, não focam tanto na modelagem do conhecimento com o objetivo de serem processadas por aplicações inteligentes.

Em Jovanovic et al. [15] é apresenta uma abordagem baseada em ontologias para anotação automática de OA e testada no Tangram, um ambiente de aprendizagem integrado para o domínio dos Sistemas de Informação Inteligentes. A abordagem fornece uma solução para geração automática de metadados para os OAs. Nesta pesquisa são apresentados os principais algoritmos de mineração de conteúdo e heurísticas aplicadas para determinar os valores de certos metadados. Especificamente, o foco é sobre os seguintes elementos: título, descrição, identificador, pessoa (com base em uma ontologia de domínio), e o papel pedagógico (com base em uma ontologia de papéis pedagógicos). Além disso, como Tangram é fundamentada em uma ontologia de estrutura de conteúdo de OA que impulsiona o processo de decomposição do OA em unidades de conteúdo é anotado o seu papel e posição na estrutura do OA. Empregando essas anotações semânticas, o TANGRAM permite montar unidades de conteúdo em novos OA personalizados para os usuários, de acordo com suas preferências e estilos de aprendizagem.

O AdaptWeb ${ }^{\circledR}$ (Ambiente de Ensino-Aprendizagem Adaptativo na Web) é um sistema e-learning adaptativo open source cujo objetivo é adaptar o conteúdo, a apresentação e a navegação de cursos na web, de acordo com o modelo do usuário/aluno. Gasparini et al. [10] detalham uma proposta de extensão do sistema AdaptWeb® para incorporar a noção de contexto e, para isso, foram desenvolvidos três serviços responsáveis por modelar as situações vivenciadas pelos estudantes, gerenciar e armazenar seus perfis e contextos e gerenciar seus objetos de aprendizagem correspondentes. O modelo apresentado em [10] visa melhorar o modelo de usuário utilizando a noção de contexto. O modelo possui três níveis de abstração: metamodelo, modelo (ontologias) e objeto. O nível metamodelo é representado por uma ontologia de topo (também chamada de nível superior), descrevendo conceitos abstratos como usuário, aplicativo, situação ou data; o nível do modelo expressa as diferentes dimensões contextuais, com diferentes ontologias para descrever os elementos que compõem o contexto e, no nível mais baixo, o modelo de objeto, onde são encontradas as instâncias das ontologias de contexto. O sistema é personalizado considerando as informações disponíveis no modelo de cada aluno. São consideradas informações do contexto pessoal, tecnológico, educacional e cultural e cada dimensão é representada por uma ontologia específica.

O trabalho de Sosnovsk et al. [25] reúne uma pesquisa sobre modelagem de usuários e ontologias web. Nele são apresentadas as tecnologias e tendências através da descrição de alguns projetos na área. O objetivo é demonstrar como desafios do desenvolvimento web nos campos da Web Adaptativa e da Web Semântica trazem novas possibilidades para a modelagem dos usuários. Segundo os autores, nos últimos anos sistemas adaptativos vem explorando ontologias com objetivos de representação semântica, aquisição automática do conhecimento, domínio e visualização do modelo do usuário e criação de soluções arquiteturais interoperáveis e reutilizáveis.

Verbert et al. [28] realizam uma ampla pesquisa a respeito de sistemas de recomendação sensíveis ao contexto no âmbito educacional. Baseando-se na análise de diversos sistemas sensíveis ao contexto implantados nos últimos anos, foram definidas categorias relevantes para elementos de contexto no âmbito educacional (Figura 1).

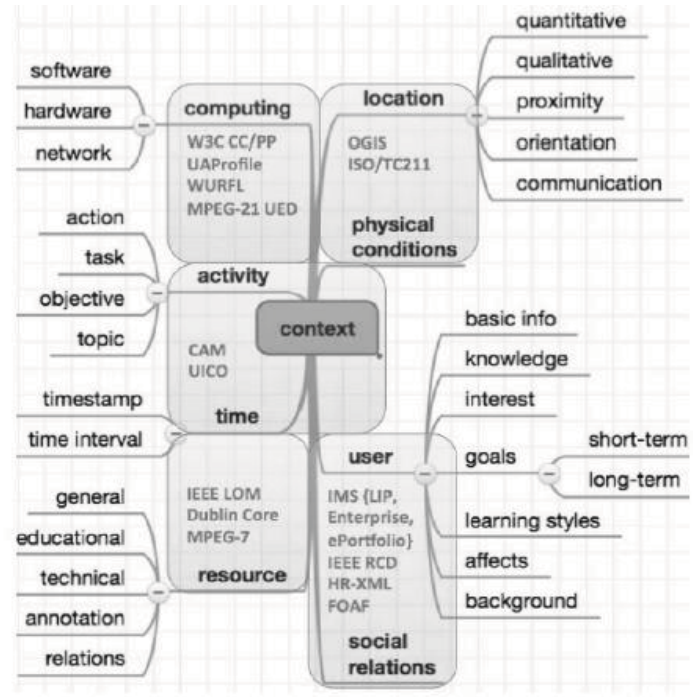

Figura 1: Framework de contexto [28]

As categorias destacadas no estudo são: espaçotemporal (localização e tempo), condições físicas, computacional, recursos (virtual e físico), usuário, atividades e social. O levantamento de como os trabalhos analisados classificam as informações mostrou que não há um consenso entre os pesquisadores em relação à definição das informações contextuais e um dos desafios futuros propostos por [28] relaciona-se ao mapeamento entre as diferentes representações de dados contextuais. A extração dos elementos de contexto e das técnicas de recomendação feita em Verbert et al. [28] tem como objetivo identificar a relevância desses elementos na recomendação de recursos. 


\subsection{Análise comparativa das propostas de recomendação}

A seguir fazemos uma breve análise das propostas descritas, a fim de identificar os termos e conceitos que irão compor a ontologia PERSONNA.

Analisando as ontologias descritas, percebe-se que elas possuem algumas características em comum, ou seja, consideram importante modelar informações estáticas (dados pessoais) e dinâmicas (objetivos, competências e preferências) do aluno. Comparando as ontologias podemos dizer que as diferenças não estão na descrição de diferentes tipos de conhecimentos, mas sim na diferença de prioridades entre os tipos de conhecimento. Isso acontece em função das diferentes perspectivas em que os autores, criadores das ontologias, iniciam sua conceitualização.

A abordagem proposta por Brusilovsky e Peylo [3] não apresenta uma ontologia específica e sim de um servidor de ontologias, que é responsável pela troca dos dados.

A ontologia proposta por Dolog e Nejdl [6] tem os termos de modelagem de dados sobre o aluno e pode ser considerada a mais completa, pois considera informações pessoais dos alunos, suas avaliações, preferências, objetivos, desempenho, certificados e portfólio. Porém ela não considera informações sobre o contexto do aluno, ponto considerado na abordagem de Niederée [18]. Nela também se verifica que o autor representa apenas os conceitos essenciais, relacionados com as preferências, desempenho, certificados e avaliações do aluno, e que os mesmos seguem os padrões PAPI [19] e LIP [14], mantendo uma correspondência direta com o domínio e terminologias existentes, satisfazendo os requisitos de clareza e legibilidade. Embora a presença de padrões restrinja a flexibilidade da descrição do modelo do aluno, isto torna a solução mais geral, pois a mesma pode ser aplicada em qualquer sistema que reconheça o padrão.

A ontologia de Dolog e Nejdl [6] também não é completa pois não considera dados relevantes como: dados pessoais do aluno, preferências, desempenho e avaliações. Os objetivos do aluno, seus estilos de aprendizagem e cognitivo não são modelados nessa ontologia. Além disso, a ontologia apenas fornece uma modelagem conceitual, não resolvendo o problema de troca dos dados entre os sistemas, mas sim dando apenas o primeiro passo, ou seja, deixando os dados em um formato padrão.

Na tabela 3 é apresentada uma sumarização das propostas analisadas, identificando quais categorias de elementos contextuais foram identificadas em cada proposta. Para categorizar os elementos de contexto extraídos das propostas, foram consideradas as categorias identificadas na pesquisa de Verbert et al. [28].

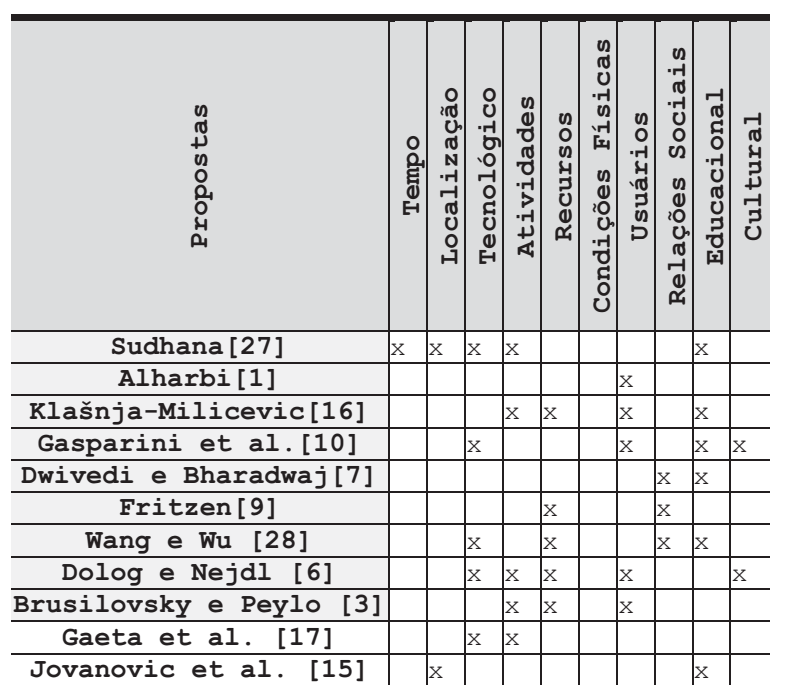

Tabela 3 - Elementos de contexto utilizados nos trabalhos relacionados.

Através da análise das propostas apresentadas foi possível perceber que a aquisição de contexto, bem como a definição dos elementos apropriados ainda é um desafio, pois muitos trabalhos utilizam elementos contextuais adquiridos através de operações manuais, como entrada de dados do usuário, apesar de alguns trabalhos já considerarem formas de aquisição automáticas. Entre os elementos contextuais utilizados foi possível observar que não há um consenso em relação à sua categorização, apesar de existir uma coincidência dos elementos usando uma nomenclatura distinta [28]. Os elementos de contexto menos explorados pelos trabalhos analisados são aqueles categorizados como condições físicas do usuário, tempo e localização.

Em geral, as propostas apresentadas dão ênfase na decomposição do modelo do aluno por características. Os autores não descrevem a metodologia utilizada para escolha dos dados contidos no modelo do aluno, apenas as classificam baseados nos sistemas que implementam.

\section{PERSONNA: uma ontologia para contexto e perfil do aluno}

Esta seção descreve a ontologia PERSONNA, que tem por objetivo servir como base para modelagem do perfil e contexto do aluno em um ambiente e-learning, a fim de padronizar as informações e facilitar o processo de recomendação de objetos de aprendizagem.

Gruber [11] descreve uma ontologia como "uma especificação explícita de uma conceitualização”, ou seja, 
uma formalização dos conceitos e relacionamentos em um domínio. Um conceito de ontologia diz respeito ao domínio modelado. Ele é utilizado para descrever uma área específica do conhecimento que será abordada na modelagem da ontologia [30]. Os benefícios de se utilizar ontologia são os mais variados: i) Possibilita um entendimento comum e compartilhado sobre conceitos em um domínio específico; ii) Permite a reutilização do domínio de conhecimento; iii) Juntamente com as linguagens baseadas em lógica descritivas, como, por exemplo, a OWL - (Web Ontology Language) e o RDF - (Resource Description Framework), permite codificar o conhecimento e a semântica para que máquinas possam compreender e iv) Torna possível o processamento do domínio por máquinas.

A ontologia representa as informações de um domínio, o que permite que sua representação possa ser utilizada por diversas pessoas, base de dados e aplicações do domínio. Assim, outras aplicações que necessitam destas informações podem utilizar essa representação, ao invés de ter que defini-las novamente.

Algumas ontologias, como nos trabalhos descritos anteriormente, têm sido definidas visando a modelagem de aluno em um contexto específico, não considerando os padrões de interoperabilidade. A ontologia proposta por Dolog e Nejdl [6] e o modelo proposto por Verbert et al.[28] são considerados os mais adequados para serem utilizadas como base da criação da ontologia PERSONNA, pois Dolog e Nejdl [6] baseiam-se nos padrões PAPI [19] e LIP [14], os quais permitem a interoperabilidade dos dados e, como resultado, tornam a solução mais geral podendo ser aplicada em qualquer sistema que reconheça esses padrões. Verbert et al. [28] apesar da pesquisa a respeito de itens de contexto e perfil de alunos, não propõem uma ontologia, relegando as possibilidades semânticas da representação. Além dos dados encontrados nestes modelos e propostas, percebeu-se a necessidade de inclusão de novos elementos relevantes a um processo de recomendação. Tais elementos foram também considerados na construção da ontologia PERSONNA.

A Tabela 4 apresenta o detalhamento da ontologia PERSONNA $^{1}$, listando suas classes, propriedades, tipos, a descrição do significado das propriedades e sua base de referência na literatura.

A ontologia PERSONNA foi especificada de forma que possa ser aderente a qualquer ambiente de aprendizagem e, para este objetivo, considerou-se os atributos mais relevantes dos alunos em relação aos modelos e padrões existentes.

${ }^{1}$ Por questões de espaço, não será apresentado o modelo gráfico da ontologia PERSONNA. No entanto, o arquivo OWL, com o detalhamento da mesma pode ser acessado em http://www.cead.ufjf.br/personna.owl
Na próxima seção é apresentada a arquitetura BROAD-RS e como a Ontologia PERSONNA foi utilizada para facilitar o processo de recomendação de objetos de aprendizagem no contexto do projeto que implementa esta arquitetura.

\section{Arquitetura BROAD-RS}

A arquitetura BROAD-RS (Figura 2), proposta em Rezende et al. [21], permite a automação do processo de recomendação de OA adaptados aos interesses do aluno com o uso de uma rede de ontologias. Este modelo também utiliza metadados retirados do padrão BROAD [4] baseado no LOM [13]. O foco desta pesquisa está na recomendação e no gerenciamento de conteúdos personalizados.

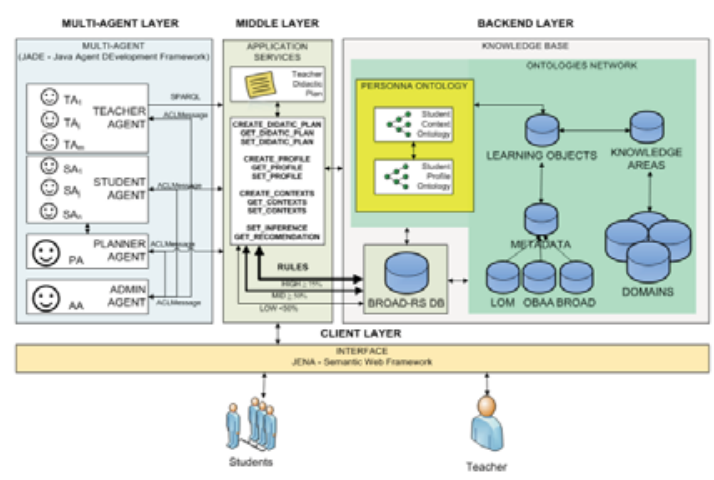

Figura 2: Arquitetura BROAD-RS.

A arquitetura está estruturada em quatro níveis: Backend layer (Nível Baixo): é a camada de conhecimento, onde se encontra a rede de ontologias e onde os OAs são armazenados. É nela que se encontram a Personna Ontology, ontologias de áreas do conhecimento e domínio e a Learning Object Ontology. A Learning Object Ontology caracteriza os recursos usando o padrão de metadados BROAD [4]. É nesta camada também que a aplicação armazena os planos didáticos e as recomendações já realizadas em um banco de dados (BROAD-RS DB), mas o perfil e contexto de aluno são representados na ontologia PERSONNA que amplia a capacidade de expressão semântica das informações de contexto e perfil do aluno.

Middle layer (Nível Médio): é a camada de serviços, utilizada para acessar as aplicações da arquitetura. É responsável pela interação dos agentes com a base de conhecimento. É nesta camada que se encontram serviços de criação, gravação e recuperação de dados de contexto, perfil e plano didático, e serviços das maquinas de inferências das ontologias.

(iii) Multi-agent layer (Nível de Agentes): esta camada 
descreve todos os agentes, seus respectivos papeis e suas inter-relações e interações com as aplicações. Os agentes relacionam os dados de contexto e perfil do aluno e plano didático do professor encontrados no banco de dados com a rede de ontologias. Como esta plataforma deve prover escalabilidade e prover recursos para a comunicação, os agentes que compõem a arquitetura estão baseados em três características básicas: reatividade (por reagir a estímulos/mensagens recebidas de outros agentes), pró-atividade (ações sem um evento, por exemplo, o agente admin monitorar a disponibilidade dos agentes) e, finalmente, a habilidade de socializar (capacidade do agente para interagir com outros agentes).

(iv) Client layer (Nível de Cliente): implementa o cadastro de objetos de aprendizagem, o cadastro de alunos e professores e a interface e telas dos usuários que pode ser desenvolvida em qualquer linguagem ou framework.

A ontologia PERSONNA foi usada na arquitetura BROAD-RS em sua rede de ontologias [5] para inferir recomendações de OA de acordo com o contexto e perfil dos alunos. Para processar estas inferências foram criadas algumas regras ontológicas e estratégias de aderência ao contexto e perfil de aprendizagem do aluno na ontologia.

\begin{tabular}{|c|c|c|c|c|}
\hline Classe & Propriedades & Tipo & Descrição & Referência \\
\hline \multirow{23}{*}{$\begin{array}{l}\text { Learner } \\
\text { Personal } \\
\text { Information } \\
\text { ( Identificação) }\end{array}$} & Name & String & Nome & {$[28][6]$} \\
\hline & Surname & String & Sobrenome & {$[28][6]$} \\
\hline & Sex & Male / Female & Sexo & {$[28][6]$} \\
\hline & Telephone & Integer & Telefone & {$[28][6]$} \\
\hline & Celphone & Integer & Telefone Celular & {$[28][6]$} \\
\hline & Email contact & String & $E-m a i l$ & {$[28][6]$} \\
\hline & Country & $\begin{array}{l}\text { Instance Data } \\
\text { Country }\end{array}$ & Pais/Nacionalidade & {$[28][6]$} \\
\hline & Postal_address & String & Endereço & {$[28][6]$} \\
\hline & Contacts & String & Informações de contato & {$[28][6]$} \\
\hline & Age & Integer & Idade & {$[28] \quad[6]$} \\
\hline & $\begin{array}{l}\text { Social_security_ } \\
\text { number }\end{array}$ & String & $\mathrm{CPF}$ & {$[28][6]$} \\
\hline & Password & String & Senha & {$[28]$} \\
\hline & Comment & String & Comentário & - \\
\hline & has_Cognitive_styles & $\begin{array}{l}\text { visual / textual } \\
\text { / auditory }\end{array}$ & $\begin{array}{l}\text { Estilo de Aprendizagem: visual, tex- } \\
\text { tual, ou auditivo }\end{array}$ & {$[28]$} \\
\hline & hasGoal & Instance Goal & Instância de Objetivos & - \\
\hline & hastime_now & Instance Time & Instância de Tempo & - \\
\hline & hasLocation_now & $\begin{array}{l}\text { Instance Loca- } \\
\text { tion }\end{array}$ & Instância de Localização & - \\
\hline & $\begin{array}{l}\text { hasphysical_- } \\
\text { conditions }\end{array}$ & $\begin{array}{l}\text { Instance Physi- } \\
\text { cal - } \\
\text { Conditions }\end{array}$ & Instância de Condição Física & - \\
\hline & hasSocial_relations & $\begin{array}{l}\text { Instance Social } \\
- \\
\text { Relations } \\
\end{array}$ & Instância de Relacionamento & - \\
\hline & hasPreferences & $\begin{array}{l}\text { Instance Prefe- } \\
\text { rences }\end{array}$ & Instância de Preferência & - \\
\hline & hasspecial_needs & $\begin{array}{l}\text { Instance Acessi- } \\
\text { bility }\end{array}$ & Instância de Condição Especial & - \\
\hline & hasinterest & InstanceInterest & Instância de Interesse & - \\
\hline & hasPerformance & $\begin{array}{l}\text { Instance Perfor- } \\
\text { mance }\end{array}$ & Instância de Desempenho & - \\
\hline \multirow{8}{*}{$\begin{array}{l}\text { Performance } \\
\text { (Desempenho) }\end{array}$} & Type & Nível do Curso & Nível do Estudo & {$[6]$} \\
\hline & Recording_date_time & DateTime & $\begin{array}{l}\text { Data-hora do registro na base de } \\
\text { dados }\end{array}$ & {$[6]$} \\
\hline & Valid_date_time_begin & DateTime & $\begin{array}{l}\text { Data e hora de registros que são } \\
\text { válidos por certos períodos de tem- } \\
\text { po, por exemplo: certificações }\end{array}$ & {$[6]$} \\
\hline & Valid_date_time_end & DateTime & $\begin{array}{l}\text { Data e hora de registros que são } \\
\text { válidos por certos períodos de tem- } \\
\text { po, por exemplo: certificações }\end{array}$ & {$[6]$} \\
\hline & Issue_from_identifier & String & Responsável pela informação & {$[6]$} \\
\hline & Issue date time & DateTime & Data e hora & {$[6]$} \\
\hline & Issue to identifier & String & Identificador & {$[6]$} \\
\hline & Learning_experience & String & Identificador da experiência do alu- & {$[6]$} \\
\hline
\end{tabular}




\begin{tabular}{|c|c|c|c|c|}
\hline & \multirow{2}{*}{$\begin{array}{l}\text { identifier } \\
\text { Competency_identifier }\end{array}$} & & \multicolumn{2}{|l|}{ no } \\
\hline & & Integer & $\begin{array}{l}\text { Identificador associado com a defi- } \\
\text { nição de competência }\end{array}$ & {$[6]$} \\
\hline & Granularity & High / Mid /Low & Granularidade & [6] \\
\hline & Coding_scheme & String & O tipo de codificação & {$[6]$} \\
\hline & Metric & String & Medidas & [6] \\
\hline & Value & String & $\begin{array}{l}\text { Nota, valor de desempenho: o grau } \\
\text { registrado Exemplo: "A", "4,3" }\end{array}$ & {$[6]$} \\
\hline \multirow{5}{*}{$\begin{array}{l}\text { Acessibility } \\
\text { (Acessibilidade) }\end{array}$} & Comment & String & $\begin{array}{l}\text { Comentários sobre a categoria (idio- } \\
\text { mas de leitura, escrita e fala do } \\
\text { aluno) }\end{array}$ & {$[28]$} \\
\hline & Capabilities & String & Lista de capacidades & {$[28]$} \\
\hline & Disabilities & String & Lista de incapacidades & {$[28]$} \\
\hline & Content_type & String & $\begin{array}{l}\text { O dado que é usado para descrever } \circ \\
\text { recurso de acessibilidade }\end{array}$ & {$[28]$} \\
\hline & Language & $\begin{array}{l}\text { Instance Data } \\
\text { Language }\end{array}$ & Idioma do recurso de acessibilidade & Novo \\
\hline \multirow{6}{*}{$\begin{array}{l}\text { Preferences } \\
\text { (Informação } \\
\text { de preferências) }\end{array}$} & Language & $\begin{array}{l}\text { Instance Data } \\
\text { Language }\end{array}$ & Idioma & {$[6]$} \\
\hline & Country & $\begin{array}{l}\text { Instance Data } \\
\text { Country }\end{array}$ & País & {$[6]$} \\
\hline & Media & $\begin{array}{l}\text { HTML / Text / } \\
\text { Video / entre } \\
\text { outros }\end{array}$ & $\begin{array}{l}\text { Lista de preferências de mídias } \\
\text { (texto, gráfico, áudio, vídeo, entre } \\
\text { outros) }\end{array}$ & {$[6]$} \\
\hline & Communicarion_tools & $\begin{array}{l}\text { Chat / Forum / } \\
\text { Email }\end{array}$ & Tipo de Comunicação Preferida & {$[6]$} \\
\hline & has Important_over & $\begin{array}{l}\text { Instance Prefe- } \\
\text { rences }\end{array}$ & Ordem de Preferência & - \\
\hline & hasDevices & Instance Device & $\begin{array}{l}\text { Lista de preferências de dispositi- } \\
\text { vos para interface homem-computador } \\
\text { (Tablets, Celular, SmartPhones, } \\
\text { PDA, PC, Notebook, TV Digital) }\end{array}$ & - \\
\hline \multirow{4}{*}{$\begin{array}{l}\text { Interest } \\
\text { (Interesses) }\end{array}$} & Type_name & String & $\begin{array}{l}\text { Tipo de interesse (hobbies, acadêmi- } \\
\text { co, profissional) }\end{array}$ & {$[28]$} \\
\hline & Comment & String & Comentários sobre a informação & {$[28]$} \\
\hline & TAG & String & Dado usado para descrever o conteúdo & {$[28]$} \\
\hline & object & String & $\begin{array}{l}\text { objeto criado como parte de uma ati- } \\
\text { vidade de interesse }\end{array}$ & {$[28]$} \\
\hline \multirow{5}{*}{$\begin{array}{l}\text { Social Relations } \\
\text { (Relacionamentos }\end{array}$} & Social_identifier & Integer & $\begin{array}{l}\text { Identificadores das pessoas que pos- } \\
\text { suem algum tipo de relacionamento } \\
\text { com o aluno }\end{array}$ & {$[28]$} \\
\hline & Relations_label & String & $\begin{array}{l}\text { Descreve a natureza do relacionamen- } \\
\text { to (amigos, pessoas, neutros, inimi- } \\
\text { gos, vizinhos, colegas de trabalho e } \\
\text { parentes) }\end{array}$ & {$[28]$} \\
\hline & Relation_to_them & String & Como está relacionado com os outros & {$[28]$} \\
\hline & Relation_to_me & String & $\begin{array}{l}\text { Como os outros estão relacionados } \\
\text { com ele }\end{array}$ & {$[28]$} \\
\hline & Relation_in_social_ne & $\begin{array}{l}\text { Instance Data - } \\
\text { social_network }\end{array}$ & $\begin{array}{l}\text { Tipo de Rede Social (Nenhuma, Face- } \\
\text { book®, Orkut }{ }^{\circledR}, \text { Twitter } ₫ \text {, Instagram }{ }^{\circledR} \text {, } \\
\text { entre outras) }\end{array}$ & - \\
\hline \multirow{5}{*}{$\begin{array}{l}\text { Time } \\
\text { ( Tempo) }\end{array}$} & Time_now & Time & Tempo Atual & {$[28]$} \\
\hline & Date_now & Date & Data Atual & {$[28]$} \\
\hline & GMT & Integer & Fuso Horário & - \\
\hline & Free_day & Boolean & Feriado & - \\
\hline & Comment & String & Comentário & - \\
\hline \multirow{9}{*}{$\begin{array}{l}\text { Device } \\
\text { (Tecnológico) } \\
\text { (Network, Softwa } \\
\text { Hardware) }\end{array}$} & Devices & $\begin{array}{l}\text { Tablets, Celu- } \\
\text { lar, } \\
\text { PC, Notebook, TV } \\
\text { Digital }\end{array}$ & $\begin{array}{l}\text { Lista de preferências de dispositi- } \\
\text { vos (Tablets, celular, PC, notebook, } \\
\text { TV Digital) }\end{array}$ & {$[28]$} \\
\hline & Type & Move / Fix & Tipo de Dispositivo (Móvel / Fixo) & [28] \\
\hline & Bandwidth & High / Low & Largura de banda (Alta, Baixa) & {$[28]$} \\
\hline & Browser & String & Navegador & {$[28]$} \\
\hline & Browser_version & Integer & Versão do Navegador & {$[28]$} \\
\hline & OS & String & Sistema Operacional & {$[28]$} \\
\hline & Resolution & String & Resolução Ex: 800x600px & {$[28]$} \\
\hline & OS Version & Integer & Versão do Sistema Operacional & {$[28]$} \\
\hline & $\mathrm{CPU}$ & String & Desempenho da CPU em $\mathrm{MHz}$ & {$[28]$} \\
\hline
\end{tabular}




\begin{tabular}{|c|c|c|c|c|}
\hline & GPU & String & Desempenho Gráfico & - \\
\hline & CODEC_list & String & Software Codec de Vídeos & - \\
\hline & Comment & String & Comentários da informação & {$[28]$} \\
\hline \multirow{8}{*}{$\begin{array}{l}\text { Goals } \\
\text { (objetivos) }\end{array}$} & Typename & $\begin{array}{l}\text { Professional / } \\
\text { Educational / } \\
\text { Personal }\end{array}$ & Nome de objetivo do aluno & {$[28][6]$} \\
\hline & Comment & String & Comentários da informação & {$[28][6]$} \\
\hline & Content_type & String & $\begin{array}{l}\text { Tipo de dado usado para descrever o } \\
\text { conteúdo }\end{array}$ & {$[28][6]$} \\
\hline & Date & Date & Datas registradas para o objetivo & {$[28][6]$} \\
\hline & Priority & High / Mid /Low & Prioridade do objetivo & {$[28][6]$} \\
\hline & Status & Boolean & Status registrado do objetivo & {$[28][6]$} \\
\hline & Description & String & Descrição do objetivo & {$[28][6]$} \\
\hline & hasSubGoals & Instance Goal & objetivos secundários & {$[28][6]$} \\
\hline \multirow{5}{*}{$\begin{array}{l}\text { Location } \\
\text { (Localização) }\end{array}$} & IP & String & IP do acesso & {$[28]$} \\
\hline & Language & $\begin{array}{l}\text { Instance Data } \\
\text { Language }\end{array}$ & Idioma do local & {$[28]$} \\
\hline & Location_country & $\begin{array}{l}\text { Instance Data } \\
\text { Country }\end{array}$ & Pais & {$[28]$} \\
\hline & Location & String & Nome do Local & {$[28]$} \\
\hline & GPS & String & $\begin{array}{l}\text { Dados de sensor Global Positioning } \\
\text { System }\end{array}$ & {$[28]$} \\
\hline \multirow{5}{*}{$\begin{array}{l}\text { PhysicalConditio } \\
\text { (Condições Físic }\end{array}$} & Heat & High / Mid /Low & Calor Ex: Alto, Médio, Baixo & {$[28]$} \\
\hline & Light & High / Mid /Low & Luz Ex: Alto, Médio, Baixo & {$[28]$} \\
\hline & Sound & High / Mid /Low & $\begin{array}{l}\text { Ruídos/Barulho Ex: Alto, Médio, Bai- } \\
\text { xo }\end{array}$ & {$[28]$} \\
\hline & Weather & String & Tempo/Clima & {$[28]$} \\
\hline & Comment & String & Comentário & \\
\hline
\end{tabular}

Tabela 4 : Categorias e Propriedades da Ontologia PERSONNA e seus detalhes.

A figura 3 apresenta algumas regras que exemplificam a utilização da ontologia PERSONNA na arquitetura BROAD-RS [21].

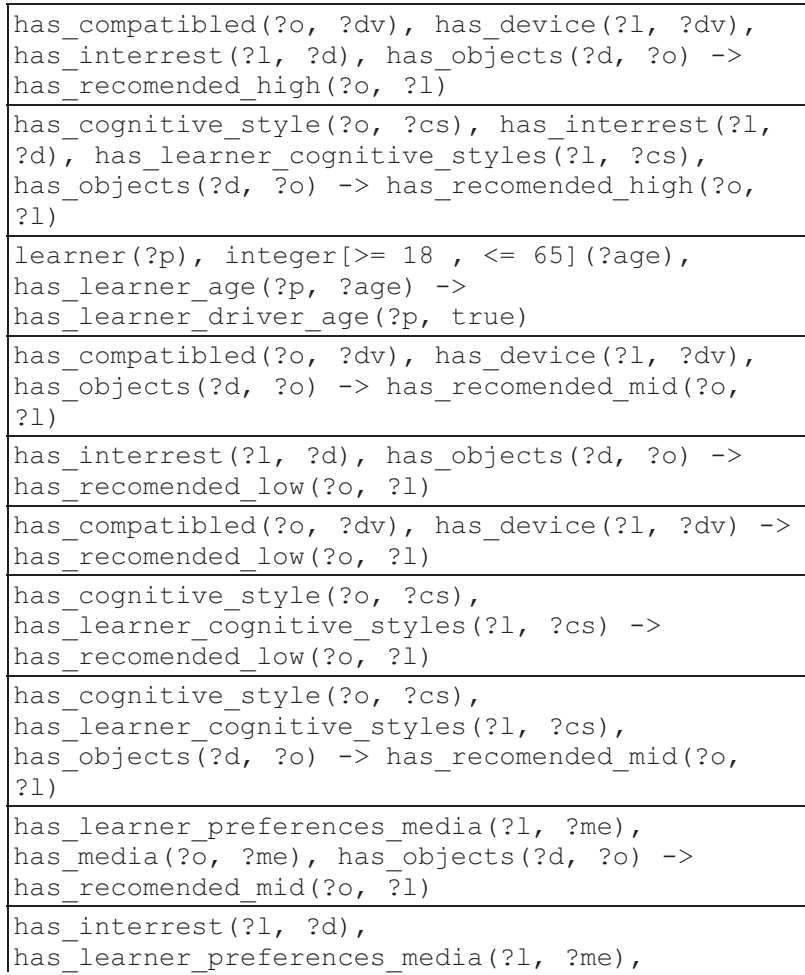

has media(?o, ?me), has objects(?d, ?o) -> has_recomended_high (?०, ?1)

Figura 3: Exemplos de Regras da Ontologia PERSONNA.

As regras, encontradas na ontologia PERSONNA e gerenciadas pelos agentes da arquitetura BROAD-RS [21], são usadas para inferir o nível de aderência do aluno a um determinado objeto de aprendizagem de acordo com seu perfil, contexto e o plano didático definido pelo professor. Para esse objetivo foram adotados três níveis:

(i) High $\geq 75 \%$ dos objetos executados atendem a um estilo de aprendizagem - totalmente aderente ao perfil.

(ii) $\quad$ Mid $\geq 50 \%$ e $<75 \%$ dos objetos executados atendem a um estilo de aprendizagem - aderente ao perfil.

(iii) Low $<50 \%$ dos objetos executados atendem a um estilo de aprendizagem - perfil não definido.

Para a ontologia PERSONNA foi necessário avaliar as porcentagens das relações entre os dados para que as regras fossem construídas (High, Mid e Low). Assim o agente Planner da arquitetura, quando necessário, executa a máquina de inferência, utilizando a ontologia. Como resultado, apresenta as recomendações necessárias para cada aluno. A Tabela 5 apresenta uma amostra das relações de porcentagem para construção de regras para ado- 
ção dos três níveis.

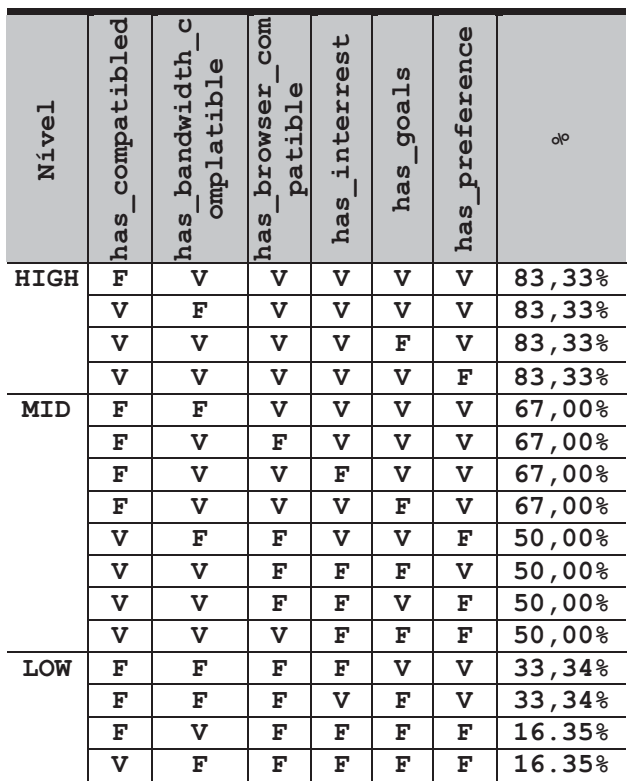

Tabela 5 : Amostra da Tabela Verdade das Regras.

Para ilustrar o uso da ontologia PERSONNA no BROAD-RS foi criado um cenário onde o aluno faz alterações de perfil e contexto no sistema e o mesmo automaticamente atualiza as recomendações de OAs (Figura 4).

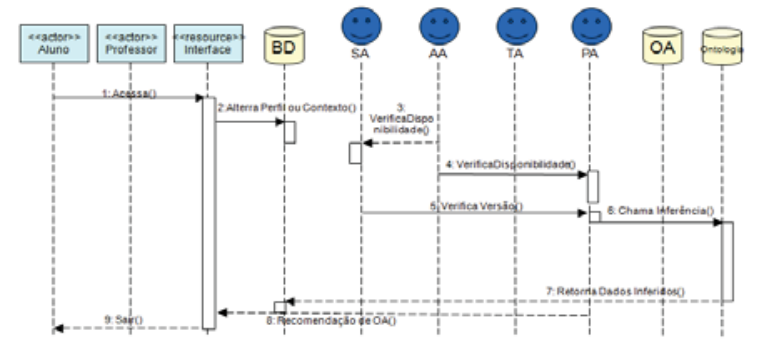

Figura 4: Cenário de Aprendizagem.

$\mathrm{O}$ usuário aluno acessa o sistema (1: Acessar()). O aluno tem a possibilidade de alterar ou criar seus dados de contexto e perfil (2: Alterar Perfil ou Contexto()). O agente AA (ADMINISTRATION AGENT) verifica se o agente SA (STUDENT AGENT) do professor e o agente PA (PLANNER AGENT) estão em modo ativo (3: VerificarDisponibilidade() e 4: VerificarDisponibilidade()). O agente SA verifica se existe atualização nos dados (5: Verificar Versão()). Como houve atualização nos dados o agente PA chama a maquina de inferência da ontologia (6: Chama Inferência()). Assim os dados inferidos são carregados em um banco de dados e apresentados na interface (7: Retorna Dados Inferidos() e (8: Recomendação de $\mathrm{OA}()))$. A figura 5 apresenta uma tela que ilustra a recomendação de objetos de aprendizagem para o aluno, considerando os dados de contexto e perfil definidos: com alta aderência (High), dois vídeos no formato MPEG, com média aderência (Mid) um vídeo em MP4 por características de seus metadados e, recomenda (Low) um artigo em formato pdf.

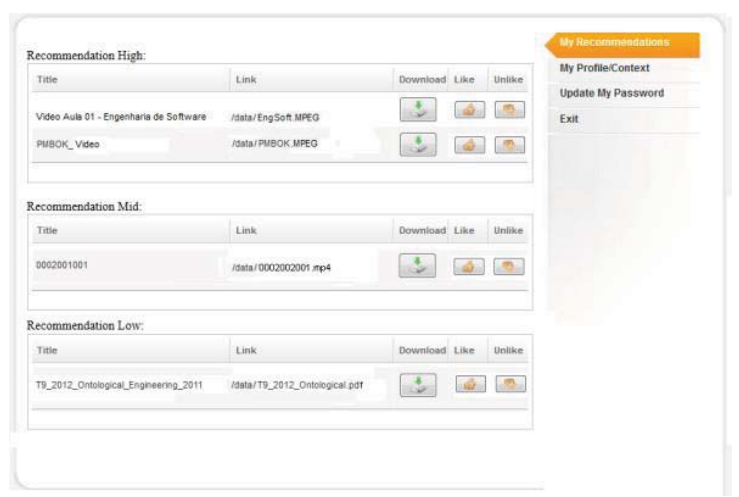

Figura 5: Tela de recomendação de OA do BROAD-RS.

\section{Considerações Finais}

Nesse artigo foram revistos trabalhos que tinham como objetivo oferecer uma solução para a padronização dos dados de alunos. Neste contexto buscou-se identificar conceitos, termos e relacionamentos que permitissem propor uma ontologia ampla e interoperável sobre o perfil e contexto de aluno para sistemas de recomendação de objetos de aprendizagem.

Através da análise de alguns modelos de aluno e, ainda, de diversas propostas de recomendação educacional que consideram o perfil e o contexto do aluno, foi possível verificar que não existe um consenso em relação às características relevantes a serem consideradas em um processo de recomendação. Diante disso, e buscando os principais elementos contextuais de cada modelo e cada proposta de recomendação, foi apresentada a Ontologia PERSONNA.

O uso de ontologias em sistemas educacionais favorece as aplicações, pois, uma ontologia formalmente definida representa a terminologia de um domínio e seu conhecimento coletivo. Além disso, a facilidade de reuso, a portabilidade e a sua disponibilidade em formato compatível com máquinas de inferência são características a serem destacadas. $\mathrm{Na}$ área educacional as ontologias veem sendo usadas em muitas aplicações de busca em repositórios de objetos de aprendizagem e sistemas de recomendação. No caso da ontologia PERSONNA a mesma foi utilizada numa aplicação de recomendação de objetos de aprendizagem onde o papel do professor é destacado.

Com a criação da ontologia PERSONNA obtivemos avanços na pesquisa de sistemas de recomendação, pois é uma ontologia mais abrangente nos dados de perfil e 
contexto de alunos. Além disso, é uma ontologia formalizada que permite a interoperabilidade dos dados e totalmente preparada para sistemas de recomendação de OA, como foi verificada e aplicada na arquitetura BROAD-RS [21]. Os resultados preliminares apontam para adequação do uso da mesma em recomendação de $\mathrm{OA}$ em cenários reais, com alunos reais.

Os próximos trabalhos do grupo estão concentrados em um Sistema de Recomendação Baseado nas Interações Sociais que pretende realizar recomendações de recursos educacionais coerentes com o perfil e o contexto do aluno, extraídos automaticamente de Sistemas Colaborativos, tais como Redes Sociais e Ambientes Virtuais de Aprendizagem [20].

Agradecimentos: à FAPEMIG, CNPq e UFJF pelo apoio financeiro ao projeto.

\section{Referências}

[1] M.T. Alharbi, A. Platt, AH AI-Bayatti. Contextaware Personal Learning Environment. The 7th International Conference for Internet Technology and Secured Transactions, 2012.

[2] R. Braga, F. Campos, F, N. Santos, et al., EFACTAGENT: Agent architecture for management, search and retrievel of e-learning artifacts, Proceedings of the IADIS International Conference on WWW/Internet Rio de Janeiro, Brazil, pp.468-472, 2011.

[3] P. Brusilovsky, C. Peylo. Adaptive and intelligent Web-based educational systems. International Journal of Artificial Intelligence in Education, 13, 2-4, Special Issue on Adaptive and Intelligent Web-based Educational Systems, 159-172, 2003.

[4] F. Campos, R. Braga, A. C. Souza et al. Projeto BROAD: Busca semântica por objetos de aprendizagem. In: ESUD 2011 - VIII Congresso Brasileiro de Ensino Superior a Distância Ouro Preto - UNIREDE, p. 3-5, Ouro Preto, Brasil, 2011.

[5] F. Campos, J. M. N. David, R. Braga et al. Rede de Ontologias: apoio semântico a linha de produtos de objetos de aprendizagem. In: Simpósio Brasileiro de Informática na Educação - SBIE, Anais do XXIII Simpósio Brasileiro de Informática na Educação, p. 3-7, Rio de Janeiro, Brasil, 2012.
[6] P. Dolog, W. Nejdl. Semantic Web Technologies for the Adaptive Web. The Adaptive Web, Springer-Verlag, Berlin, Heidelberg , pp. $697-$ 719, 2007.

[7] P. Dwivedi, K.K. Bharadwaj. E-learning recommender system for learners in online social networks through association retrieval, In: Proceedings of the CUBE International Information Technology Conference, 6(1), pp. 676-681, 2012.

[8] EDUPERSON. EduPerson Object Class Specification (200210). Disponível em: $<$ http://middleware.internet2.edu/eduperson>. 2005, Acesso em: 29 de agosto 2013.

[9] E. Fritzen, S. W. M. Siqueira, L.C.V. Andrade. Recuperação Contextual de Informação na Web para Apoiar Aprendizagem Colaborativa em Redes Sociais. In: Simpósio Brasileiro de Informática na Educação, 2012, Rio de Janeiro. Anais do Simpósio Brasileiro de Informática na Educação. Porto Alegre: SBC, 2012.

[10] I. Gasparini, A. Kemczinski, M.S. Pimenta et al. Modelo do usuário sensível ao contexto cultural em um sistema e-learning adaptativo. Informática na Educação (Impresso), v. 14, p. 123-135, 2011.

[11] T. A. Grumber. Translation Approach to Portable Ontology Specifications. 1993. Disponível em: $<$ http://tomgruber.org/writing/ontolingua-kaj1993.pdf.> Acesso em: 01 de setembro 2013.

[12] V. T. Hinz, I. Gasparini, M. S. Pimenta. Em busca de recomendação de objetos de aprendizagem em um ambiente e-learning com uso de ontologias - In: LACLO 2011 - Sexto Congresso Latinoamericano de Objetos de Aprendizagem, Montevidéu. LACLO 2011 Sexto Congresso Latinoamericano de Objetos de Aprendizagem, 2011.

[13] IEEE, Draft Standard for Learning Object Metadata. Disponível em: http://ltsc.ieee.org/wg12/files/LOM_1484_12_1_ v1_Final_Draft.pdf., 2002.

[14] IMS LIP. IMS Learner Information Packaging Information Model Specification. Disponível em:

http://www.imsglobal.org/profiles/lipinfo01.html , 2007. Acesso em: 01 de setembro 2013. 
[15] J. Jovanovic, D. Gasevic V. Devedzic. Ontology-Based Automatic Annotation of Learning Content. International Journal on Semantic Web and Information Systems (IJSWIS), 2(2), 91-119. doi:10.4018/jswis.2006040103, 2006.

[16] A. Klašnja-Milicevic, B. Vesin, M. Ivanovi et al. Integration of recommendations and adaptive hypermedia into java tutoring system, Computer Science and Information Systems, 8(1), pp. 211224, 2011.

[17] M. Gaeta; F. Orciuoli; P. Ritrovato. Advanced ontology management system for personalised eLearning. Know.-Based Syst. 22, 4 (May 2009), 292-301. DOI=10.1016/j.knosys.2009.01.006 http://dx.doi.org/10.1016/j.knosys.2009.01.006, 2009.

[18] A. Niederée A. et al. Ontologically Enriched User Profiling for Cross System Personalization. In: User Modelling, Edinburgh. Proceedings [S.1.]: ACM Press. 2005.

[19] PAPI. IEEE P1484.2/d7, 2001. Draft standard for learning technology. Public and Private information para learners. Disponível em: $<$ ltsc.ieee.org/wg1/files/ltsa 06.doc / $>$. Acesso em: Acesso em: 30 de agosto 2013.

[20] C.K. Pereira, F. Campos, V. Ströele et al. Extração de Características de Perfil e de Contexto em Redes Sociais para Recomendação de Recursos Educacionais. In: Simpósio Brasileiro de Informática na Educação, 2014, Dourados-MS. Anais do Simpósio Brasileiro de Informática na Educação pp 506-515. Porto Alegre: SBC, 2014.

[21] Rezende, P.; Campos, F.; David, J. M. N.; Braga, R. BROAD-RS: uma arquitetura para recomendação de objetos de aprendizagem sensível ao contexto usando agentes, Anais do ESUD 2013 - X Congresso Brasileiro de Ensino Superior a Distância - UNIREDE, Belém/PA, Brasil, junho, 2013.

[22] SABA. Universal Learner Format - ULF Technical Specification Version 1.0 (2000)
Disponível em: $<$ http://xml.coverpages.org/ulfSpecification 2000 1204.pdf > . Acesso em: 29 de agosto 2013.

[23] N. Santos, F. C. Campos, R. Braga. Digital Libraries and Ontology. In: Yin-Leng Theng; Schubert Foo; Dion Hoe-Lian Goh; Jin-Cheon Na.. (Org.). Handbook of Research on Digital Libraries: Design, Development, and Impact. Hershey, Pensilvania: Idea Group Inc, v. 1, pp. 206-214, 2008.

[24] J. B. Schafer, J. Konstan, J. Riedl. Recommender systems in e-commerce. In: ACM Conference on Eletronic Commerce, Proceeding Denver: ACM. Denver, Colorado, EUA,p. 158-166, 1999.

[25] S. Sosnovsky, D. Dicheva. Ontological technologies for user modelling. Int. J. Metadata Semant. Ontologies 5, 1 (April 2010), 32-71. DOI=10.1504/IJMSO.2010.032649 http://dx.doi.org/10.1504/IJMSO.2010.032649, 2010 .

[26] A. C. Souza, F. Camps, R. Braga. Metadados Educacionais para Objetos de Aprendizagem: Projeto BROAD. In: Congreso Iberoamericano de Informática Educativa, Santiago. Anais do RIBIE, v. 1. pp. 653-659, 2010.

[27] K. M. Sudhana. An Ontology-based Framework for Context-aware Adaptive E-learning System. International Conference on Computer Communication and Informatics (ICCCI -2013), Jan. 04 - 06, 2013, Coimbatore, India, 2013.

[28] K. Verbert, N. Manouselis, X. Ochoa et al. Context-Aware Recommender Systems for Learning: A Survey and Future Challenges, IEEE Transactions on Learning Technologies, vol. 99, no. PrePrints, 2012.

[29] S. Wang, C. Wu. Application of context-aware and personalized recommendation to implement an adaptive ubiquitous learning system, Expert Systems with Applications 38 (2011) pp. 1083110838, 2011.

[30] L. Yu. A Developers Guide to the Semantic Web. Atlanta: Springer, cap 2, 4 e 5, 201 